\title{
Bridging the gap between Ox and Gauss using OxGauss
}

Citation for published version (APA):

Laurent, S., \& Urbain, J. R. Y. J. (2004). Bridging the gap between Ox and Gauss using OxGauss. METEOR, Maastricht University School of Business and Economics. METEOR Research Memorandum No. 005 https://doi.org/10.26481/umamet.2004005

Document status and date:

Published: 01/01/2004

DOI:

10.26481/umamet.2004005

Document Version:

Publisher's PDF, also known as Version of record

\section{Please check the document version of this publication:}

- A submitted manuscript is the version of the article upon submission and before peer-review. There can be important differences between the submitted version and the official published version of record.

People interested in the research are advised to contact the author for the final version of the publication, or visit the DOI to the publisher's website.

- The final author version and the galley proof are versions of the publication after peer review.

- The final published version features the final layout of the paper including the volume, issue and page numbers.

Link to publication

\footnotetext{
General rights Owners
rights.

- You may freely distribute the URL identifying the publication in the public portal. please follow below link for the End User Agreement:

www.umlib.nl/taverne-license

Take down policy

If you believe that this document breaches copyright please contact us at:

repository@maastrichtuniversity.nl

providing details and we will investigate your claim.
}

Copyright and moral rights for the publications made accessible in the public portal are retained by the authors and/or other copyright owners and it is a condition of accessing publications that users recognise and abide by the legal requirements associated with these

- Users may download and print one copy of any publication from the public portal for the purpose of private study or research.

- You may not further distribute the material or use it for any profit-making activity or commercial gain

If the publication is distributed under the terms of Article $25 \mathrm{fa}$ of the Dutch Copyright Act, indicated by the "Taverne" license above, 


\title{
Bridging the Gap Between Ox and Gauss using OxGauss
}

\author{
Sébastien Laurent \\ CeReFim (Université de Namur) \\ and CORE (Université catholique de Louvain). \\ E-mail: Sebastien.Laurent@fundp.ac.be. \\ Correspondence to CeReFim, 8 rempart de la vierge, B5000 Namur, Belgium. \\ Phone: +32 (0) 81724869 . \\ Fax: +32 (0) 81724840 . \\ and \\ Jean-Pierre Urbain \\ Department of Quantitative Economics, University Maastricht, \\ The Netherlands. E-mail: j.urbain@ke.unimaas.nl.
}

First Draft: October 2003

This version: January 2004

\section{Introduction}

The purpose of this paper is to review and discuss the key improvements brought to OxGauss, a program that comes with modern versions of Ox. ${ }^{1}$ OxGauss provides a way to run Gauss ${ }^{2}$ code under Ox or to call an existing Gauss procedure under Ox in a similar way than one can call $\mathrm{C}$ (dll) or fortran codes from Gauss and Ox. Unlike the old g2ox program that came with earlier versions of Ox, OxGauss is not a translator from Gauss to Ox.

Depending on the goal of the analysis and on the experience of the user, both use can prove to be particularly useful. From an Ox user point of view, the main objective of OxGauss is probably to allow the many existing Gauss codes to be called from Ox with only a minimum number of changes

\footnotetext{
${ }^{1}$ There are two versions of Ox. Oxconsole can be downloaded from http://www.nuff.ox.ac.uk/Users/Doornik/, which is the main Ox web page. The console version is free for educational purposes and academic research. The Professional Windows version, or commercial version comes with a nice interface for graphics known as GiveWin (available for purchase from Timberlake Consultants, http://www.timberlake.co.uk).

${ }^{2}$ Gauss is sold by Aptech Systems, 23804 S.E. Kent-Kangley Rd., Maple Valley, WA, 98038, USA; see http://www.aptech.com/.
} 
to these codes. This is beneficial to both the Ox user and the writer of the Gauss codes as it increases the visibility and hence the potential use of the underlying statistical technique. This may also help in the transition from Gauss to $\mathrm{Ox}$ if this is the purpose of the exercise.

On the other hand, running a pure Gauss code with OxGauss is attractive for the non-Gauss and potentially even non-Ox users in that it makes the replication of published work possible using the console version of Ox. It has considerable merit since the replicability of simulation and empirical results in econometrics is recognized as being a fairly important aspect of research as exemplified by the practice of the Journal of Applied Econometrics that asks authors to make their data and possibly specialized codes available to the potentially interested reader. Not surprisingly, an increasing number of researchers in econometrics are making their codes and routines freely available to the econometrics community. For this reason, OxGauss also fulfils a genuine need in that it provides the researcher with a free and rather simple solution to run Gauss codes.

The paper is structured as follows: in Section 2, we propose an overview of OxGauss and give some simple examples as well as a speed comparison between Ox, OxGauss and Gauss 3.5. Section 3 discusses the graphical issue. Section 4 proposes to test the usefulness of OxGauss in replicating the results of a broad number of research papers. Finally, Section 5 concludes.

\section{OxGauss}

As explained above, the purpose of OxGauss is twofold: calling Gauss codes from Ox and running Gauss codes without having to install Gauss. The next two subsections illustrate these two features of OxGauss.

\subsection{Calling Gauss codes from $O x$}

The main objective of OxGauss is probably to allow Gauss code to be called from Ox. This helps in the transition to Ox, and increases the amount of code that is available to users of Ox.

In order to illustrate how Gauss codes can be called from OX, we consider a small project that mixes both Gauss and Ox codes. The first file, Gaussprocs.src, consists of a code file containing the procedure gengarch(omega,alpha,beta,nu,T_0,T,n) that simulates a GARCH model. This procedure has been written by Dick van Dijk (see Franses and van Dijk, 2000) and is downloadable from his web site http://www. few.eur.nl/few/people/djvandijk/nltsmef/nltsmef.htm.

To call this procedure from an Ox code, one first has to create a header file. The purpose of the header file is simply to allow the declaration of the functions, constants and external variables to be known wherever it is required. The reason is that, to avoid a compilation error in Ox, any function or global variable has to be explicitly declared before its use. In our example, the header file (Gaussprocs.h) consists of the following instructions: 


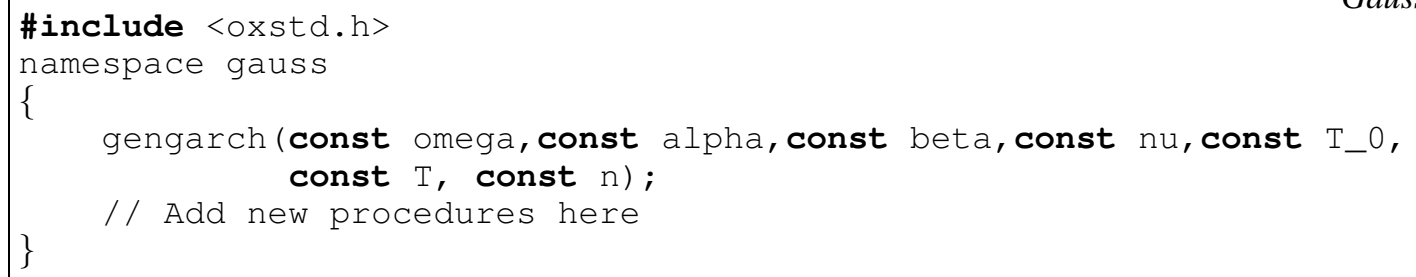

Additional procedures can be added in Gaussprocs.src but the header file has to be modified accordingly. ${ }^{3}$ It is recommended to use the.$s r c$ extension for the Gauss programs and.$h$ for the header files.

In the example GarchEstim.ox, we use the Gauss procedure to generate 20.000 observations following a GARCH $(1,1)$ process with Student-t errors and then, rely on the Ox package G@RCH 3.0 (see Laurent and Peters, 2002) to estimate a GARCH(1,1) model by gaussian Quasi-Maximum likelihood. To do so, the OxGauss code must be imported into the Ox program, together with the G@RCH package. The \#import command has been extended to recognize OxGauss imports by prefixing the file name with gauss: : .

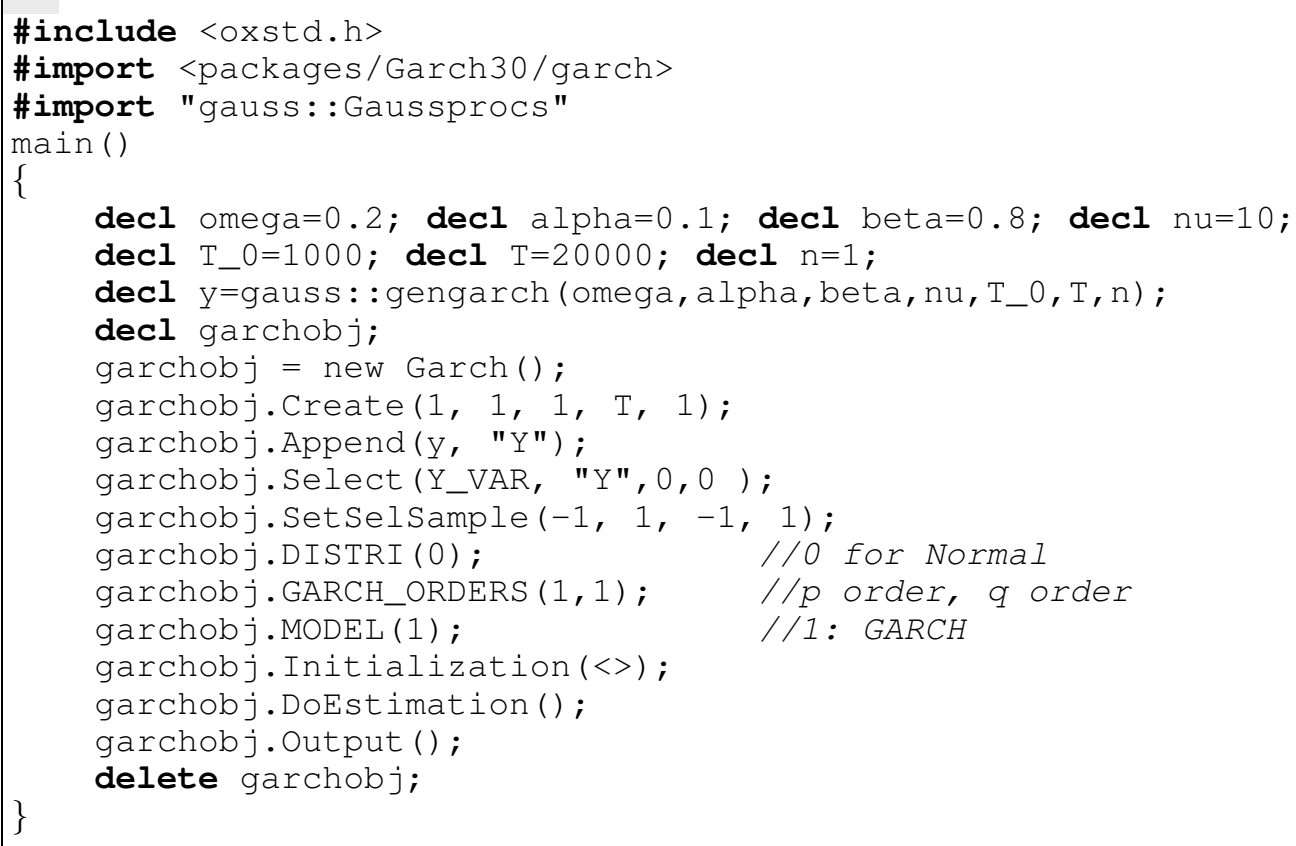

Note that when the OxGauss functions or variables are accessed, they must also be prefixed with the identifier gauss: : .

To run this program on the command line, the user simply has to enter oxl GarchEstim.ox. Alternatively, it can be launched from OxEdit. OxEdit 1.62 (or later) is a free but powerful text editor for Windows provided with both versions of Ox 3.3. Like GiveWin, OxEdit supports syntax colouring

\footnotetext{
${ }^{3}$ Arguments declared const can be referenced, but cannot be changed inside the function.
} 
of Ox programs, and context-sensitive help. The first time OxEdit is used, the user should execute the Preferences/Add Predefined Modules menu and select Ox. Ox and Gauss programs can then be run from the Modules menu without leaving OxEdit. See also the OxEdit web page http://www.oxedit.com for more details. Finally, users of the Ox Professional can run Ox codes within GiveWin by using the menu Modules/Start OxRun.

\subsection{Running Gauss codes}

The second potentially attractive feature of OxGauss is to enable the user to directly run a wide range of Gauss programs under Ox.

As an example, we consider the Gauss package Mixed Logit Estimation Routine for Panel Data of Kenneth Train, David Revelt and Paul Ruud. The archive file train0299.zip (available at http://elsa.berkeley.edu/Software/abstracts/train0296.html) contains seven files including the code file mxlp.g and the data. This program has been written by Kenneth Train and used by this author in a collection of papers (see the web site above for more details) dealing with mixed logit models. ${ }^{4}$

In order to save space, we do not report the 1396 lines of code of the main file mxlp.g. This program can be run on the command line by entering oxl $-g \mathrm{mxlp}$.g. Alternatively, it can be launched from OxEdit (Modules/OxGauss menu) or within GiveWin by using the menu Modules/Start OxGauss. Note that while the previous versions of OxGauss required a few modifications on the codes, ${ }^{5}$ the program is now almost fully compatible with the new version. The only problem is that the program estimates the model by maximum likelihood, giving the opportunity to the user to chose the maximization routine domax of Paul Ruud or the commercial package maxlik. Launching the program could lead to the following error message:

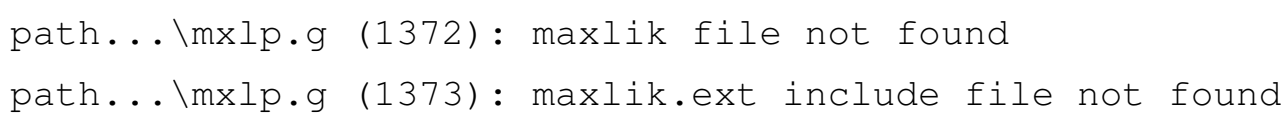

To solve this problem one can either comment out lines 421 to 451 concerning the add-on maxlik (and use the domax procedure, i.e. the default option $O P T I M=1$ in the program) or install the M@ximize package used in Section 4. As expected, the results are very similar, if not identical (the only difference is detected after the sixth decimal of the standard errors).

\footnotetext{
${ }^{4}$ Mixed logit (also called random-parameters logit) generalizes standard logit by allowing the parameter associated with each observed variable (e.g., its coefficient) to vary randomly across units (e.g. individuals or customers).

${ }^{5}$ On his web site (http://facweb.arch.ohio-state.edu/pviton/support/oxgauss), Philip Viton mentioned about 6 operations to make under Ox 3.2 before succeeding to run the code without compilation error.
} 


\subsection{Understanding OxGauss}

When an OxGauss program is run, it automatically includes the / include/oxgauss . ox file. This itself imports the required files: ${ }^{6}$

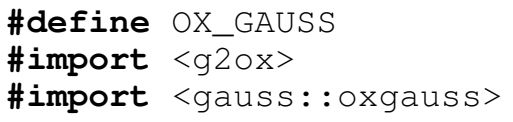

These import statements ensure that $\mathrm{g} 2 \mathrm{ox} . \mathrm{h}$ and oxgauss. $\mathrm{h}$ are being included. The majority of the OxGauss run-time system is in \include $\operatorname{g} 20 x .0 x$ while the keywords are largely in oxgauss.src.

A nice feature of OxGauss is its transparency since most of the codes that link Gauss functions to Ox are gathered in the file $\backslash$ include $\backslash \mathrm{g} 20 \mathrm{x} .0 \mathrm{x}$. For instance, the output of the Gauss function cumprodc (x) is a $N \times K$ matrix with the cumulative products of the columns of the $N \times K$ matrix $x$. The Ox code here below (copy from the file $\mathrm{g} 2 \mathrm{ox} . \mathrm{ox}$ ) shows how OxGauss interprets this function.

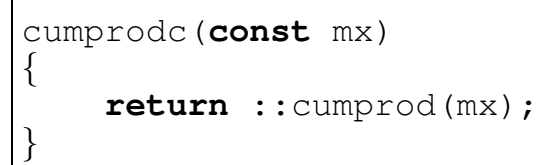

From this example, it is clear from that OxGauss does not translate the Gauss code into Ox but makes the link between the Gauss function (here cumprodc) and its Ox counterpart (cumprod). When the corresponding Ox function does not exist, an ox code is written between the brackets, aiming at computing the same thing as the original Gauss function. It is important to note all the Gauss functions are not supported by OxGauss. For instance, there is no equivalent of the Gauss function intgrat2 (for the computation of double integrals) in Ox 3.3. For this reason, the corresponding procedure in g20x.ox just reports the error message intgrat2 () unsupported (see below). However, if such a function becomes available in a next version of Ox, mapping ingrat 2 to the corresponding function in Ox will be a child's play!

Tables A1 and A2 of the appendix (available on the web site of the JAE Data Archive) give a list of all the Gauss functions supported by OxGauss. To simplify the reading of the list, we report precompiled functions (or directly mapped functions) like sin in Table A1 and open source functions (like cumprodc, see above) in Table A2. Adding adding all the functions leads to a total of 420 functions recognized by OxGauss. Table A3 in the appendix gives a list of 64 Gauss functions not supported by the current version of Ox (or about 15\%).

\footnotetext{
${ }^{6}$ For ease of presentation, the filename is printed in the upper right corner of the window.
} 


\subsection{Speed Comparison}

As pointed out by Cribari-Neto (1997), the main strength of Ox is its speed, although Gauss performs quite well too and its speed performance are not far behind those of Ox. A recent and detailed comparison between several mathematical programs performed by Stefan Steinhaus (see http://www.scientificweb.de/ncrunch/) shows that Ox is the winner in terms of speed. Since OxGauss just implements a layer on Ox, OxGauss is expected to be slower than Ox. But one may wonder how slower is it and how it really compares to Gauss in terms of speed? To answer these two questions we consider the Benchmark tests proposed by Stefan Steinhaus (edition 3). Note that since the functions intquad 2 and intquad 3 (double and triple integration of functions) are not available in Ox 3.3, the corresponding tests have been discarded which leads a total of 14 points of comparison. To perform the speed comparison, we did first execute the Ox benchmark program Benchox 2 .ox with 5 replications of each test on a Pentium III $450 \mathrm{Mhz}$ and $526 \mathrm{MB}$ RAM running under Windows 98 with Windows versions of the programs. We also conducted the same experiment with the Gauss benchmark program Benchga2 .prg using OxGauss, Gauss 3.5. The results are reported in Table 1.

Table 1 Speed Comparison (timing in seconds) between Ox 3.3, OxGauss and Gauss 3.5.

\begin{tabular}{lrrr}
\hline \hline Operation & Ox 3.3 & OxGauss & Gauss 3.5 \\
\hline Creation, trans. \& reshaping of a 1000x1000 matrix: & 1.043 & 1.153 & 1.043 \\
1000x1000 random matrix to the power 1000: & 1.023 & 1.003 & 1.083 \\
Sorting of 2,000,000 random values: & 9.190 & 9.577 & 9.643 \\
FFT over 1,048,576 random values: & 4.777 & 5.423 & 5.417 \\
Determinant of a 1000x1000 random matrix: & 14.590 & 14.593 & 14.593 \\
Creation of an 1400x1400 Toeplitz matrix: & 0.167 & 0.167 & 0.200 \\
Inverse of a 1000x1000 random matrix: & 36.433 & 36.600 & 36.930 \\
Eigenvalues of a 600x600 random matrix: & 35.573 & 35.867 & 36.563 \\
Choleski decomposition of a 1000x1000 random matrix: & 4.360 & 4.380 & 4.437 \\
Creation of 1000x1000 cross-product matrix: & 8.953 & 12.817 & 12.777 \\
Calculation of 500000 fibonacci numbers: & 1.377 & 1.380 & 1.423 \\
Gamma function on a 1000x1000 random matrix: & 0.737 & 0.763 & 0.730 \\
Gaussian error function over a 1000x1000 random matrix: & 0.930 & 0.950 & 0.790 \\
Linear regression over a 1000x1000 random matrix: & 28.563 & 28.597 & 28.713 \\
\hline Ben
\end{tabular}

Benchmark programs run (5 replications of each test) on a Pentium III 450 Mhz.

As a whole, we see from this table that OxGauss compares very well to Gauss 3.5 in terms of speed. Indeed, while Ox is in general faster than OxGauss and Gauss 3.5 (when taking the 14 experiments we see that Ox is about $4 \%$ faster than OxGauss and Gauss 3.5), the difference between OxGauss and Gauss 3.5 is very small (OxGauss is found to be on average about $0.5 \%$ faster than 
Gauss 3.5). ${ }^{7}$

\section{Graphs using GnuDraw}

While most graphical features of Gauss are recognized by OxGauss via Ox Professional for Windows (through GiveWin), Oxconsole has no graphs support. Nevertheless, the user of the console version can rely on the Ox package GnuDraw developed by Charles Bos that allows the creation of GnuPlot (see http://www.gnuplot.info) graphics from Ox. The package is free of charge and is downloadable from his homepage http://www.tinbergen.nl/ cbos/, together with the GnuPlot software. Note that a detailed help file gnudraw.html and a few example codes are provided with the package, which makes its use very friendly.

A nice feature of this package is its platform independence (unlike the current version of GiveWin which is only available for Windows) which provide non-Windows users (e.g. Unix, Linux, Solaris, Sun, etc.) an efficient graph support. When using Ox 3.30, GnuPlot can be called automatically from within Ox. Usage of GnuDraw is intended to be simple - see Cribari-Neto and Zarkos (2003) for a comprehensive overview of the GnuDraw package. Interestingly, as OxGauss implements just a layer over Ox, it is possible to instruct the underlying Gauss to call GnuDraw routines instead of the OxDraw routines. The program gnuGauss.prg implements this.

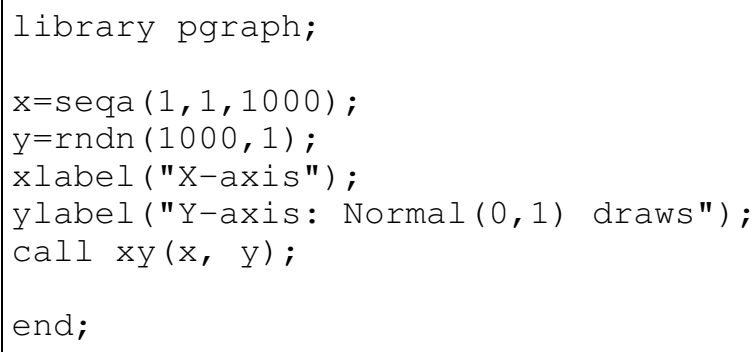

\section{Replicating empirical results using OxGauss}

The main drawback of OxGauss until now is that it was not suited to run Gauss codes that make use of commercial applications. However, an interesting characteristic of OxGauss is that it is extensible. The GnuDraw package presented here above is a first example of additional package extending OxGauss.

For instance OxGauss reports an error message if the Gauss code requires one of the optimizators from the modules Cml, Maxlik, or Optmum (see Section 2.2). This makes OxGauss useless in many

\footnotetext{
${ }^{7}$ While a comparison with a more recent version of Gauss is of potential interest, we do not investigate this issue since the speed comparison is not the aim of the paper. Note that we have done the same experiment with Gauss 3.2.29 and the result is that Gauss 3.5 is a much improved release in terms of speed.
} 
situations of practical interest, ... except if we provide to Oxgauss an additional package that bridges the gap between these three libraries and the optimizators of Ox.

To test the feasibility of this task, we have written a set of procedure gathered in a package called M@ximize 1.0. By means of transparency, the package is open source and freely available on the web at the following address: http://www.core.ucl.ac.be/ laurent.

It is important to note first that it is not our intention to review our package here. Indeed, the main goal of this section is to test the reliability OxGauss in a non-trivial situation. Second, the M@ximize package does not translate the Gauss optimizators into Ox as we just link the options; nor to clone the optimizators. Indeed, in the first release of the package (M@ximize 1.0) not all the functions of these optimizators are available, although the most important options of Cml, Maxlik, and Optmum are taken into account.

As explained above, the main reason for using OxGauss is probably to replicate the results obtained in a research paper. To test OxGauss in a real-life situation, we have downloaded from the internet a huge number of Gauss codes. Here is a list of five web sites that we have visited from which both the data and the Gauss codes can be retrieved:

James Hamilton: http://weber. ucsd.edu/〜 jhamilto/

Bruce Hansen: http: //www.ssc.wisc.edu/〜bhansen/

Chang-Jin Kim: http://www.econ.washington.edu/user/cnelson/SSMARKOV.htm

Luc Bauwens: http://www.core.ucl.ac.be/econometrics/bauwens.htm

Rolf Tschering: http://www.personeel.unimaas.nl/r.tschernig/

We have also used the codes provided by Kim and Nelson (1999) in their book on markovswitching models (Chapters 3 to 11). Table A4 in the appendix gives the list of papers that we have replicated (i.e. 27 references). Most of these papers rely quite heavily on non-linear optimization techniques and thus require one the three above mentioned optimizators of Gauss. Consequently, to run these codes under OxGauss one need first to install the $\mathrm{M} @$ ximize package (i.e. by unzipping the file m@ximize.zip in the main directory of Ox).

While most of the codes can be run in their present form, some marginal changes in the GAUSS codes are sometimes needed. The most frequently encountered problems are:

- Converting data files. For instance, running the Gauss code related to the reference 22 in Table A4 gives the Invalid .FMT or .DAT file error message. The reason is that old style Gauss data sets (v89 .dht/.dat) must be converted to the new Gauss format (v96 .dat). The program to do this conversion is ox/lib/dht2dat. The conversion can be run from the command line as:

oxl lib/dht2dat old_datafile.dht new_datafile.dat

Alternatively, the data files can be converted to the new format through GiveWin by loading first the.$d h t$ file and second saving the file into the new format. 
- Absence of extension. To launch a Gauss code using OxEdit, the file needs an extension. It is common to use the extension.src.

- Interactive mode. Examples 1, 6, 9 and 13 use the Gauss function cons that requests an input from the keyboard (console) and puts it into a string. The typical use of this function is to generate a message like "Do you wish to continue (y or $\mathrm{n}$ )?". Depending of the result the program takes one direction or the another. In other words, the program enters in an interactive mode. In such a case the program has to be launched using "Ox interactive", i.e. Oxli.exe under Windows instead of Oxl.exe. ${ }^{8}$

As illustration, we consider the Gauss package written by Rolf Tschernig accompanying the paper of Yang and Tschernig (1999) published in the Journal of the Royal Statistical Society, Series B (reference 27 in Table A4). We focus on the example file provided by the author, i.e. multband.tes that estimates the asymptotic optimal vector bandwidth for simulated bivariate non-linear regression models. This file is made up of about 190 lines of Gauss code and includes three libraries, i.e. Optmum, pgraph and multband (a library provided by the author) as well as a set of three dll files. To use the package under Gauss, one has first to install the library multband by first copying the file multband.lcg into the subdirectory . / lib of Gauss and second the files multband.src (about 2900 lines of code) and multband.dec (declarations of global variables) into the subdirectory . / src. Finally, to complete the installation, one has to copy the three dll files locling.dll, density.dll and loccubg.dll into the subdirectory . / d lib. Importantly, to use the package under OxGauss, one has to follow the same instructions and copy the files into the existing subdirectories . / OxGauss/lib, . / OxGauss/src and . /OxGauss/dib.

Now the example file multband.tes can be executed. ${ }^{9}$ Once again, the results are identical (up to the sixth decimal). The outputs obtained with OxGauss and Gauss 3.2 are reported in Table A5 (in the appendix).

\section{Conclusion}

This paper presents a review and a discussion of OxGauss, an application that enables the user to run a wide range of Gauss programs/codes under Ox without needing to have Gauss installed on his/her machine. One main drawback of OxGauss is that it is of little use once the purpose is to execute a program that requires one of the three well know Gauss application modules Cml, Maxlik or Optmum.

\footnotetext{
${ }^{8}$ When using OxEdit to run the Gauss code, an additional shortcut has to be created. The simple solution is to click on the menu View/Preferences/Add/Remove Modules. Then clone the OxGauss shortcut and in the Command line change Oxl.exe by Oxli.exe.

${ }^{9}$ Notice that this example file simulates a sequence of 250 observations. To make the comparison between Gauss and OxGauss possible we have changed the original code that now always uses (load) the same random numbers.
} 
In this paper we show that since OxGauss is extensible, it is possible to fix this problem by writing a set of additional procedures that bridge the gap between Ox and the above mentioned optimizers.

It is important to emphasize that OxGauss is potentially useful both for Gauss and Ox users. On the one hand Gauss codes can efficiently be called under Ox. This means that Ox programmers willing to use existing Gauss procedures do not have to translate the procedures into Ox but can call the Gauss code directly under Ox. On the other hand, OxGauss can be used to run the Gauss code(s) under Ox and hence to replicate the results of a paper for which Gauss code is made available by the author(s). The effectiveness of OxGauss is illustrated by revisiting a large number of Gauss codes that are freely available on the internet and that use Gauss application modules that require numerical optimization (26 papers published in international reviews and the procedures related to a book, see Table A4). Importantly in all cases the programs were found to be fully compatible with OxGauss in the sense that no change was required (or very minor changes) on the original code and that the results were almost identical.

To conclude, we believe that OxGauss could bring the Gauss and Ox user communities closer. We could even hope that Gauss users who already share their Gauss codes would start testing the compatibility with OxGauss, make the changes to ensure compatibility if necessary and indicate that their code is "OxGauss compliant".

\section{Acknowledgment}

While remaining responsible for any errors in this paper, the authors would like to thank the editor

James G. MacKinnon and Jean-Philippe Peters for useful comments and Charles Bos and Jurgen Doornik for their accessibility.

\section{References}

CRIBARI-Neto, F. (1997): “Econometric Programming Environments: Gauss, Ox and S-Plus,” Journal of Applied Econometrics, 12, 77-89.

Cribari-Neto, F., and S. Zarkos (2003): "Econometric and Statistical Computing Using Ox," Computational Economics, 21, 277-295.

Franses, P., and D. VAN DiJK (2000): Non-Linear Series Models in Empirical Finance. Cambridge University Press.

KIM, C.-J., and C. Nelson (1999): State-Space Models with Regime Switching: Classical and Gibbs-Sampling Approaches with Applications. The MIT Press.

Laurent, S., and J.-P. Peters (2002): “G@RCH 2.2 : An Ox Package for Estimating and Forecasting Various ARCH Models," Journal of Economic Surveys, 16, 447-485. 
Appendix of

Bridging the Gap Between Ox and Gauss using OxGauss

\author{
Sébastien Laurent \\ CeReFim (Université de Namur) \\ and CORE (Université catholique de Louvain). \\ E-mail: Sebastien.Laurent@fundp.ac.be. \\ Correspondence to CeReFim, 8 rempart de la vierge, B5000 Namur, Belgium. \\ Phone: +32 (0) 81724869 . \\ Fax: +32 (0) 81724840 . \\ and \\ Jean-Pierre Urbain \\ Department of Quantitative Economics, University Maastricht, \\ The Netherlands. E-mail: j.urbain@ke.unimaas.nl.
}

First Draft: October 2003

This version: January 2004 
Table A1: Precompiled functions supported by OxGauss

\begin{tabular}{lllll}
\hline fcmptol & delete & lib library & output & save \\
abs & det & library & outwidth n & saveall \\
arccos & diagrv & line & pdfn & screen \\
arcsin & disable & ln & plot x,y & scroll \\
arctan & dlibrary & load x & plotsym n & shell \\
arctan2 & dllcall & loadf f & pqgwin & show \\
atan & enable & loadk k & prcsn n & sin \\
atan2 & end/stop & loadm x & print & sinh \\
cdfchic & erf & loadp p & printdos str & sqrt \\
cdfchii & errorlog str & loads s & rank & system \\
cdffc & exp & locate m,n & replay & tan \\
cdfn & eye & log & rerun & tanh \\
cdfnc & feq & lowmat & rev & toeplitz \\
cdfni & floor & lprint & rndcon c & trace new \\
cdftc & fmod & lpwidth n & rndmod m & trap new \\
ceil & format & lshow & rndmult a & trunc \\
cols & gamma & meanc & rndn & use gcgfile \\
cos & graph & median & rndseed seed & vcx \\
cosh & hsec & msym str & rndu & vech \\
create & inv & new & round & xpnd \\
datalist & invpd & ones & rows & zeros \\
debug & ismiss & open & run filename &
\end{tabular}


Table A2: Open source functions supported by OxGauss

\begin{tabular}{|c|c|c|c|c|c|c|}
\hline balance & corrvc & etstr & keyw & polymult & seekr & upper \\
\hline band & corrx & exctsmpl & lag1 & polyroot & selif & utrisol \\
\hline bandchol & counts & exec & lagn & printfm & seqa & vals \\
\hline bandcholsol & countwts & export & lncdfbvn & printfmt & seqm & $\mathrm{vcm}$ \\
\hline bandltsol & crossprd & exportf & lncdfn & prodc & setcnvrt & vec \\
\hline bandrv & crout & fcheckerr & $\operatorname{lncdfn} 2$ & putf & setdif & vecr \\
\hline bandsolpd & croutp & fclearerr & lncdfnc & qnewton & setvmode & vget \\
\hline base10 & csrtype & fflush & $\operatorname{lnfact}$ & qprog & shiftr & wait \\
\hline besselj & cumprodc & $\mathrm{fft}$ & lnpdfmvn & qqr & sleep & waitc \\
\hline bessely & cumsumc & ffti & lnpdfn & qqre & solpd & writer \\
\hline cdfbeta & cvtos & fftn & loadd & qqrep & sortc & xpnd \\
\hline cdfbvn & date & fge & lower & $\mathrm{qr}$ & sortcc & \\
\hline cdfchinc & datestr & fgets & lowmat1 & qre & sorthc & \\
\hline cdffnc & datestring & fgetsa & ltrisol & qrep & sorthcc & \\
\hline cdfgam & datestrymd & fgetsat & lu & qrsol & sortind & \\
\hline cdfn2 & dayinyr & fgetst & lusol & qrtsol & sortindc & \\
\hline cdftci & delif & fgt & $\operatorname{maxc}$ & qtyr & sortmc & \\
\hline cdftnc & design & files & maxindc & qtyre & sqpsolve & \\
\hline cdftvn & detl & fle & maxvec & qtyrep & stof & \\
\hline cdir & dfft & flt & mbesselei & quantile & stop & \\
\hline changedir & dffti & fne & mbesselei0 & qyr & strindx & \\
\hline chol & dfree & fopen & mbesselei1 & qyre & strlen & \\
\hline choldn & diag & formatcv & mbesseli & qyrep & strput & \\
\hline cholsol & dos & formatnv & mbesseli0 & rankindx & strrindx & \\
\hline cholup & dotfeq & fputs & mbesseli1 & readr & strsect & \\
\hline chrs & dotfge & fputst & meanc & real & submat & \\
\hline close & dotfgt & fseek & $\operatorname{minc}$ & recode & subscat & \\
\hline closeall & dotfle & fstrerror & minindc & recserar & substute & \\
\hline & dotflt & ftell & miss & recsercp & sumc & \\
\hline cmadd & dotfne & ftocv & missex & recserrc & svd & \\
\hline cmcplx & dstat & ftos & missrv & $\mathrm{rfft}$ & svd1 & \\
\hline cmcplx2 & dummy & gammaii & moment & rffti & $\operatorname{svd} 2$ & \\
\hline cmdiv & dummybr & gausset & ndpchk & rfftip & svdcusv & \\
\hline cmemult & dummydn & getf & ndpclex & rfftn & svds & \\
\hline cmimag & eig & getname & ndpcntrl & rfftnp & svdusv & \\
\hline cminv & eigh & gradp & null1 & rfftp & sysstate & \\
\hline cmmult & eighv & hasimag & ols & rndbeta & system & \\
\hline cmreal & eigrg & hessp & olsqr & rndgam & tab & \\
\hline cmsoln & eigrg2 & imag & olsqr2 & rndnb & tempname & \\
\hline cmsub & eigrs & import & orth & rndns & time & \\
\hline cmtrans & eigrs2 & indcv & packr & rndp & timestr & \\
\hline code & eigv & indexcat & parse & rndus & token & \\
\hline color & end & indices & pause & rndvm & trapchk & \\
\hline colsf & envget & indices 2 & pi & rotater & trim & \\
\hline con & eof & indnv & pinv & rowsf & trimr & \\
\hline cond & eqsolve & intrsect & polychar & rref & type & \\
\hline cons & erfc & invswp & polyeval & save & union & \\
\hline conv & error & iscplx & polyint & saved & uniqindx & \\
\hline coreleft & etdays & iscplxf & polymake & scalerr & unique & \\
\hline corrm & ethsec & key & polymat & scalmiss & upmat & \\
\hline
\end{tabular}


Table A3: Functions not supported by OxGauss (under Ox 3.3)

\begin{tabular}{lllll}
\hline cdfbvn2 & filesa & makevars & sortd & vnamecv \\
cdfbvn2e & getnr & medit & spline1d & vput \\
cdfmvn & getpath & mergeby & spline2d & vread \\
complex & header & mergevar & stdc & vtypecv \\
conj & hess & momentd & tocart & \\
csrcol & importf & nametype & topolar & \\
csrlin & intgrat2 & nextn & typecv & \\
editm & intgrat3 & nextnevn & typef & \\
eigcg & intquad1 & null & varget & \\
eigcg2 & intquad2 & optn & vargetl & \\
eigch & intquad3 & optnevn & varput & \\
eigch2 & intrleav & quantiled & varputl & \\
fftm & intsimp & schtoc & vartype & \\
fftmi & lncdfbvn2 & schur & vartypef & \\
fileinfo & lncdfmvn & setvars & vlist &
\end{tabular}


Table A4: List of codes associated to papers

\footnotetext{
1. HAMILTON, J. (1994): State-Space Models, in Handbook of Econometrics, Volume 4, 3039-3080, edited

Hamilton, J. (1996): “The Daily Market for Federal Funds”, Journal of Political Economy, pp. 26-56.

Hamilton, J. (1996): "Specification Testing in Markov-Switching Time-Series Models", Journal of Econometrics, 70, 127-157.

HAMilton, J., and C. Engle (1990): "Long Swings in the Exchange Rate: Are They in the Data and Do Markets Know It?", American Economic Review, pp. 689-713.

HAMILTON, J., and O. JORDA (2002): "A Model for the Federal Funds Rate Target", Journal of Political Economy, 110, 1135-1167.

HAMilton, J., and G. LiN (1996): "Stock Market Volatility and the Business Cycle", Journal of Applied Econometrics, 11, 573-593.

Hamilton, J., and G. Perez-Quiros (1996): "What Do the Leading Indicators Lead?", Journal of Business, 69, $27-49$.

HAmilton, J., and R. Susmel (1994): "Autoregressive Conditional Heteroskedasticity and Changes in Regime", Journal of Econometrics, 64, 307-333.

Bauwens, L. M. Lubrano (1998): Bayesian Inference on GARCH models using the Gibbs Sampler, The Econometrics Journal, 1, C23-C46.

Hansen, B. (1992): "Tests for Parameter Instability in Regressions with I(1) Processes", Journal of Business and Economic Statistics, 10, 321-335.

Hansen, B. (1992): "Testing for Parameter Instability in Linear Models", Journal of Policy Modeling, 14, 517-533.

Hansen, B. (1992): "The likelihood Ratio Test under Non-standard Conditions: Testing the Markov Switching Model of GNP”, Journal of Applied Econometrics, 7, S61-S82.

Hansen, B. (1994): “Autoregressive Conditional Density Estimation”, International Economic Review, 35, 705-730.

Hansen, B. (1996): "Inference when a Nuisance Parameter is not Identified under the Null Hypothesis", Econometrica, 64, 413-430.

Hansen, B. and A. Gregory (1996): "Residual-based Tests for Cointegration in Models with Regime Shifts", Journal of Econometrics, 70, 99-126.

Hansen, B. (1997): "Approximate Asymptotic p-values for Structural Change Tests", Journal of Business and Economic Statistics, $15,60-67$.

Hansen, B. (1997): "Inference in TAR Models", Studies in Nonlinear Dynamics and Econometrics, 2, 1-14.

Hansen, B. (1999): "Testing for Linearity", Journal of Economic Surveys, 13, 551-576.

Hansen, B. (2000): "Sample Splitting and Threshold Estimation", Econometrica, 68, 575-603.

Hansen, B (2000): "Testing for Structural Change in Conditional Models", Journal of Econometrics, 97, 93-115.

Hansen, B. and M. Caner (2000): "Threshold Autoregression with a Unit Root", Econometrica, 69, 1555-1596.

Hansen, B., D. Cox and E. Jimenez: "How Responsive are Private Transfers to Income? Evidence from a Laissez-faire Economy", forthcoming in Journal of the Public Economics. Hansen, B. and B. Seo (2002): "Testing for Threshold Cointegration", Journal of Econometrics, 110, 293-318.

Hansen, B. (2001): "The New Econometrics of Structural Change: Dating Changes in U.S. Labor Productivity", Journal of Economic Perspectives, 15, 117-128.

Hansen, B.: "Recounts from Undervotes: Evidence from the 2000 Presidential Election", forthcoming in Journal of the American Statistical Association.

Kim, C.-J. and C. Nelson (1999): State-Space Models with Regime Switching: Classical and Gibbs-Sampling Approaches with Applications, The MIT Press.

27. Yang, L. and R. Tschernig (1999): “Multivariate Bandwidth Selection for Local Linear Regression”, Journal of the Royal Statistical Society, Series B, 61, 793-815.
} 
Table A5: Outputs obtained by running multband.tes (see reference 27 in Table A4) under OxGauss (left) and Gauss 3.2 (right)

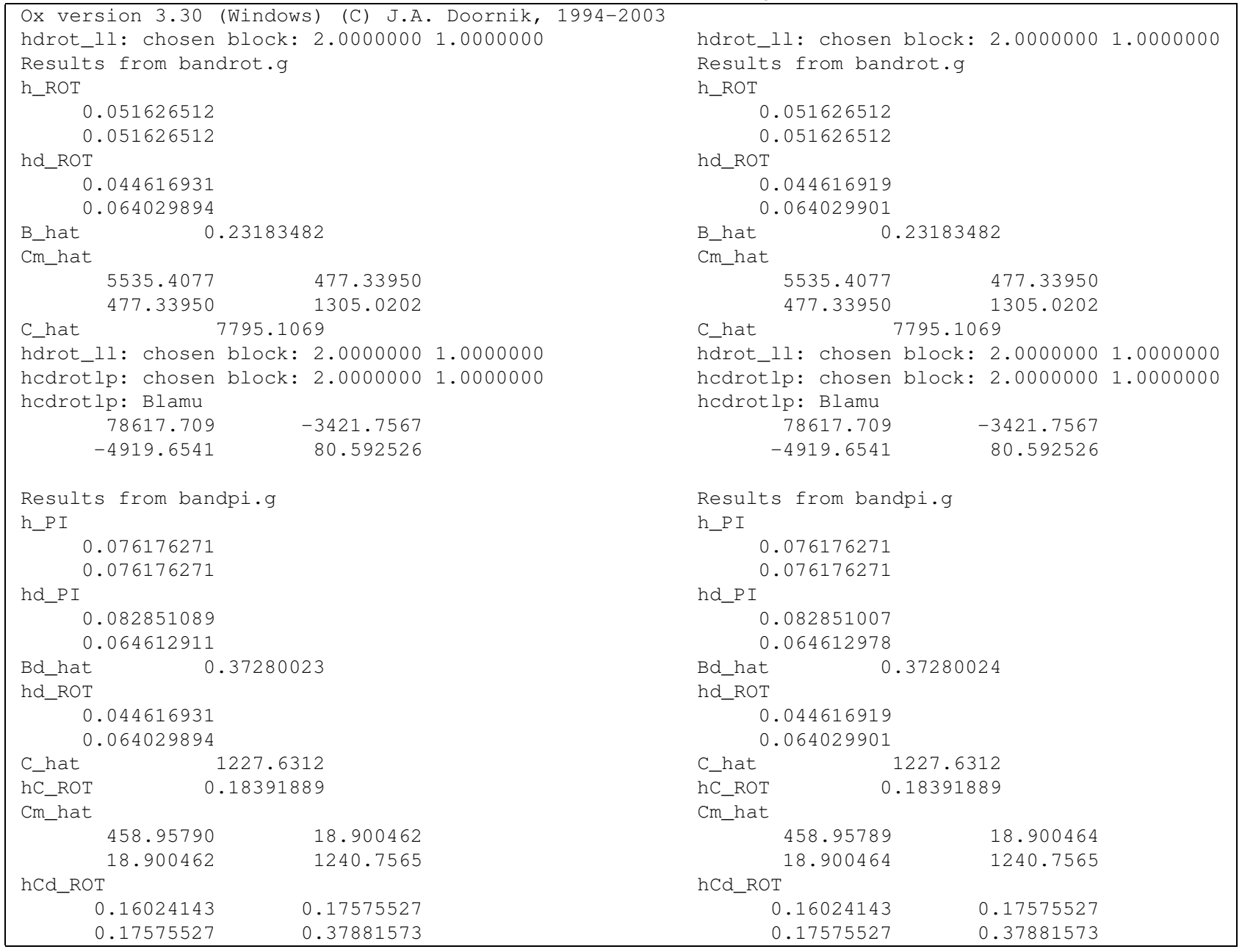

